ESAIM: PROCEEDINGS AND SURVEYS, November 2014, Vol. 46, p. 146-162

ECIT 2012 - Witold Jarczyk, Daniele Fournier-Prunaret, João Manuel Gonçalves Cabral

\title{
APPLICATION OF DIMENSIONED NUMBERS TO FUNCTIONAL EQUATIONS
}

\author{
HIDEAKI IZUMI ${ }^{1}$
}

\begin{abstract}
In this paper, we introduce the notion of dimensioned numbers and extended dimensioned numbers. The notion of dimensioned numbers originates in geometric measurements, and it can be used to describe iterated exponential functions of a single variable. We apply the theory of dimensioned numbers to solving some functional equations of a single variable.
\end{abstract}

Résumé. Dans cet article, nous introduisons la notion de nombres dimensionnés et prolongés nombres dimensionnés. La notion de nombres dimensionnés vient de mesures géométriques, et ils peuvent être utilisés pour représenter les fonctions exponentielles répétées d'une seule variable. Nous appliquons la théorie des nombres dimensionnés à la résolution de certaines équations fonctionnelles d'une seule variable.

\section{INTRODUCTION}

Let us consider to express geometric quantities with their dimensions. For example, we denote two points by $2_{0}$, line segment of length 5 by $5_{1}$, rectangle of area 8 by $8_{2}$ and cube of volume 10 by $10_{3}$. We can use multiplication to single out the area of rectangle or the volume of cuboid. The area of rectangle with sides of length $a$ and $b$ is $a b$ :

$$
a_{1} \times b_{1}=a b_{2},
$$

and the volume of cuboid with base area $c$ and height $d$ is $c d$ :

$$
c_{2} \times d_{1}=c d_{3} .
$$

Moreover, quantities of the same dimension are addable. For example, by concatenating two line segments of length $a$ and $b$, we get length $a+b$ :

$$
a_{1}+b_{1}=(a+b)_{1},
$$

but no calculations are carried out for the addition of quantities of different dimensions.

From the calculations above it is natural to define a multiplication and an addition of quantities with general dimension by

$$
\begin{aligned}
a_{c} \cdot b_{d} & =a b_{c+d}, \\
a_{c}+b_{c} & =(a+b)_{c} .
\end{aligned}
$$

\footnotetext{
${ }^{1}$ Chiba Institute of Technology, Shibazono 2-1-1, Narashino, Chiba, 275-0023, Japan

e-mail: izumi.hideaki@it-chiba.ac.jp
} 
Next, we observe binary operations between monomials of variable $x$ :

$$
\begin{aligned}
a x^{c} \cdot b x^{d} & =a b x^{c+d}, \\
a x^{c}+b x^{c} & =(a+b) x^{c} .
\end{aligned}
$$

The similarity between (1) and (3), and between (2) and (4) is obvious. In fact, numbers with dimension are models of polynomials of a single variable. We call them dimensioned numbers. Moreover, we also consider dimensioned numbers where its dimension itself is a dimensioned number, e.g.,

$$
2_{3_{4}}, \quad 5_{-1_{8_{0.2}}} \cdot
$$

These numbers are considered to correspond to the functions

$$
2 x^{3 x^{4}}, \quad 5 x^{-x^{8 x^{0.2}}}
$$

namely, iterated exponentials.

In this paper, we will develop the theory of abstract dimensioned numbers, and establish the correspondence between dimensioned numbers and iterated exponential functions of a single variable. After that, we introduce the notion of extended dimensioned numbers, in which we can deal with both infinite power series and infinitely iterated exponentials. Finally, as an application of dimensioned numbers, we will obtain formal solutions to several types of functional equations. In this paper, we will search only for monic solutions, namely solutions of the form

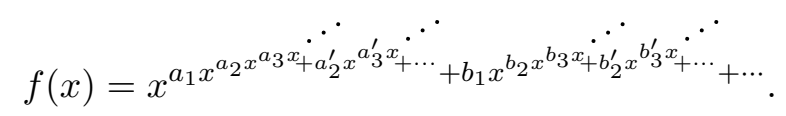

For other types of formal solutions to functional equations, like exponential series or trigonometric series, see the author's paper [Iz1] as well as his presentation [Iz2].

\section{Abstract dimensioned numbers}

We will construct abstract dimensioned numbers by induction on their depth, which corresponds to how many times we need to repeat iteration of exponentials to get the function. Dimensioned numbers of depth $n$ is naturally embedded into those of depth $n+1$, so that we obtain an inductive system. Moreover, we will introduce a binary operation called "symmetric powers" to dimensioned numbers.

Depth 0: We set

$$
\mathbb{D}_{0}:=\mathbb{R}=(\mathbb{R},+, 0, \cdot, 1,>),
$$

namely, dimensioned numbers of depth 0 are just real numbers equipped with the usual ordered ring structure: addition + , zero element 0 , multiplication $\cdot$, unit element 1 and the linear order $>$ having the properties

(a1) if $a, b, c \in \mathbb{R}$ and $a>b$, then $a+c>b+c$, and

(a2) if $a, b \in \mathbb{R}, a>0$ and $b>0$, then $a b>0$.

Depth 1: First, we define $\boldsymbol{D}_{1}$ as the free abelian group generated by symbols $a_{b}, a \in \mathbb{R}, b \in \mathbb{D}_{0} ;$ namely,

$$
\boldsymbol{D}_{1}:=\left\{\begin{array}{l|l}
a_{b^{(1)}}^{(1)}+a_{b^{(2)}}^{(2)}+\cdots+a_{b^{(m)}}^{(m)} & \begin{array}{c}
a^{(1)}, a^{(2)}, \cdots, a^{(m)} \in \mathbb{R}, \\
b^{(1)}, b^{(2)}, \cdots, b^{(m)} \in \mathbb{D}_{0}
\end{array}
\end{array}\right\} \cup\{0\}
$$

where 0 is the zero element of this abelian group. We define a product $\cdot$ in the set $\left\{a_{b} \mid a \in \mathbb{R}, b \in \mathbb{D}_{0}\right\}$ by

$$
a_{b} \cdot c_{d}:=a c_{b+d}, a, c \in \mathbb{R}, b, d \in \mathbb{D}_{0}
$$


and extend them to whole $\boldsymbol{D}_{1}$ by using the distributive law. For example,

$$
\left(a_{b}+a_{b^{\prime}}^{\prime}\right) \cdot c_{d}=a_{b} \cdot c_{d}+a_{b^{\prime}}^{\prime} \cdot c_{d}=a c_{b+d}+a^{\prime} c_{b^{\prime}+d} .
$$

The element $1_{0}$ is the unit element for the multiplication:

$$
1_{0} \cdot a_{b}=a_{b} \cdot 1_{0}=a_{b},
$$

and the element 0 is indeed the zero element for the multiplication:

$$
0 \cdot A=\left(1_{0}-1_{0}\right) \cdot A=1_{0} \cdot A-1_{0} \cdot A=A-A=0, A \in \boldsymbol{D}_{1} .
$$

It is easy to see the following lemma.

Lemma 1.1. $\left(\boldsymbol{D}_{1},+, 0, \cdot 1_{0}\right)$ is a commutative ring.

To realize the equality (2) in $\boldsymbol{D}_{1}$, we define an ideal in $\boldsymbol{D}_{1}$. Let $\boldsymbol{Z}_{1}$ be the additive subgroup of $\boldsymbol{D}_{1}$ generated by the elements in the set

$$
\left\{a_{b}+a_{b}^{\prime}-\left(a+a^{\prime}\right)_{b} \mid a, a^{\prime} \in \mathbb{R}, b \in \mathbb{D}_{0}\right\} \cup\left\{0_{b} \mid b \in \mathbb{D}_{0}\right\} .
$$

Lemma 1.2. $\boldsymbol{Z}_{1}$ is an ideal in $\boldsymbol{D}_{1}$.

Proof. Let $c \in \mathbb{R}, d \in \boldsymbol{D}_{1}$. Then we have

$$
\begin{aligned}
& c_{d} \cdot\left\{a_{b}+a_{b}^{\prime}-\left(a+a^{\prime}\right)_{b}\right\}=a c_{b+d}+a^{\prime} c_{b+d}-\left(a c+a^{\prime} c\right)_{b+d} \in Z_{1}, \\
& c_{d} \cdot 0_{b}=0_{b+d} \in Z_{1} .
\end{aligned}
$$

Hence $\boldsymbol{Z}_{1}$ is an ideal in $\boldsymbol{D}_{1}$.

We define

$$
\mathbb{D}_{1}:=\boldsymbol{D}_{1} / \boldsymbol{Z}_{1}
$$

We will always identify an element $A$ in $\boldsymbol{D}_{1}$ with the element $A+\boldsymbol{Z}_{1}$ in the residue ring $\mathbb{D}_{1}$. We call elements in $\mathbb{D}_{1}$ dimensioned numbers of depth 1 . Note that elements of the form $0_{b}, b \in \mathbb{R}$, is equal to 0 . Since

$$
\left(a_{b^{(1)}}^{(1)}+a_{b^{(2)}}^{(2)}+\cdots+a_{b^{(m)}}^{(m)}\right)+\left(\left(-a^{(1)}\right)_{b^{(1)}}+\left(-a^{(2)}\right)_{b^{(2)}}+\cdots+\left(-a^{(m)}\right)_{b^{(m)}}\right)=0_{b^{(1)}}+0_{b^{(2)}}+\cdots+0_{b^{(m)}}=0,
$$

the additional inverse of $a_{b^{(1)}}^{(1)}+a_{b^{(2)}}^{(2)}+\cdots+a_{b^{(m)}}^{(m)}$ is $\left(-a^{(1)}\right)_{b^{(1)}}+\left(-a^{(2)}\right)_{b^{(2)}}+\cdots+\left(-a^{(m)}\right)_{b^{(m)}}$, namely,

$$
-\left(a_{b^{(1)}}^{(1)}+a_{b^{(2)}}^{(2)}+\cdots+a_{b^{(m)}}^{(m)}\right)=\left(-a^{(1)}\right)_{b^{(1)}}+\left(-a^{(2)}\right)_{b^{(2)}}+\cdots+\left(-a^{(m)}\right)_{b^{(m)}} .
$$

For a dimensioned number

$$
A=a_{b^{(1)}}^{(1)}+a_{b^{(2)}}^{(2)}+\cdots+a_{b^{(m)}}^{(m)} \in \mathbb{D}_{1},
$$

we call $a^{(1)}, a^{(2)}, \cdots$ heads of $A$ and $b^{(1)}, b^{(2)}, \cdots$ dimensions of $A$. We say a dimensioned number $A$ is simple if $A$ is of the form

$$
A=a_{b}
$$

namely, containing only one head. Otherwise, $A$ is called a compound dimensioned number. If the head of a simple dimensioned number $A$ is 1 , namely,

then we call $A$ monic.

$$
A=1_{b},
$$


Next, we will introduce a linear order in $\boldsymbol{D}_{1}$. Let

$$
A=a_{b^{(1)}}^{(1)}+a_{b^{(2)}}^{(2)}+\cdots+a_{b^{(m)}}^{(m)} \in D_{1}
$$

be a non-zero element. Without loss of generality, we may assume

$$
a^{(1)} \neq 0, a^{(2)} \neq 0, \ldots a^{(m)} \neq 0 \text { and } b^{(1)}>b^{(2)}>\cdots>b^{(m)} .
$$

Then we call $a_{b^{(1)}}^{(1)}$ the dominant term of $A$. We define the sign of $A$ according to that of the head of the dominant term:

$$
\begin{aligned}
& A>0(A \text { is positive }) \quad \Longleftrightarrow a^{(1)}>0, \text { and } \\
& A<0(A \text { is negative }) \Longleftrightarrow a^{(1)}<0,
\end{aligned}
$$

and define a relation $>$ in $\mathbb{D}_{1}$ by

$$
A>B \Longleftrightarrow A-B>0, A, B \in \mathbb{D}_{1}
$$

Lemma 1.3. $\left(\mathbb{D}_{1},+, 0, \cdot 1_{0},>\right)$ is an ordered integral domain.

Proof. Since $\mathbb{D}_{1}$ is a residue ring of $\boldsymbol{D}_{1}, \mathbb{D}_{1}$ is a commutative ring.

We will show that $>$ is a linear order in $\mathbb{D}_{1}$. Let $A, B, C \in \mathbb{D}_{1}$ and suppose $A>B$ and $B>C$. Denote the dominant terms of $A-B$ and $B-C$ by $a_{b}$ and $a_{b^{\prime}}^{\prime}$ respectively. From the assumption, we have $a>0$ and $a^{\prime}>0$. Since $A-C=(A-B)+(B-C)$, the dominant term of $A-C$ is either $a_{b}$ or $a_{b^{\prime}}^{\prime}$ if $b \neq b^{\prime}$ and $\left(a+a^{\prime}\right)_{b}$ if $b=b^{\prime}$. In either case we have $A>C$. So the transitivity holds. For any $A, B \in \mathbb{D}_{1}$, it is clear that exactly one among $A>B, A=B, A<B$ holds. So $>$ is a linear order in $\mathbb{D}_{1}$.

Next, we ascertain the following two conditions

(b1) if $a, b, c \in \mathbb{D}_{1}$ and $a>b$, then $a+c>b+c$, and

(b2) if $a, b \in \mathbb{D}_{1}, a>0$ and $b>0$, then $a b>0$.

The condition (b1) is clear, so we will examine (b2). Let $A, B \in \mathbb{D}_{1}$ and suppose that $A>0$, $B>0$. We denote the dominant terms of $A$ and $B$ by $a_{b}$ and $a_{b^{\prime}}^{\prime}$ respectively. From the assumption, we have $a>0$ and $a^{\prime}>0$. Then by expanding the product $A B$ by using the distributive law, we see that the dominant term of $A B$ is $a a_{b b^{\prime}}^{\prime}$. Since $a a^{\prime}>0$, we have $A B>0$. Hence (b2) holds.

Finally, we will show that $\mathbb{D}_{1}$ is an integral domain. Let $A, B$ be non-zero elements in $\mathbb{D}_{1}$. We may assume that both $A$ and $B$ are positive, replacing $A$ or $B$ by $-A$ or $-B$ respectively, if necessary. Then from (b2) we see that $A B>0$. Hence $A B \neq 0$. So $\mathbb{D}_{1}$ is an integral domain.

Next, we will define a map $i_{0}^{1}: \mathbb{D}_{0} \rightarrow \mathbb{D}_{1}$ by

$$
i_{0}^{1}(a):=a_{0}, a \in \mathbb{D}_{0}
$$

It is easy to see that $i_{0}^{1}$ is an injective ordered ring homomorphism.

Depth $n(n=2,3, \ldots)$ : Suppose that the ring $\mathbb{D}_{n-1}$ of dimension numbers of depth $n-1$ is constructed. We define $\boldsymbol{D}_{n}$ as the free abelian group generated by symbols $a_{B}, a \in \mathbb{R}, B \in \mathbb{D}_{n-1}$. Hence,

$$
\boldsymbol{D}_{n}:=\left\{\begin{array}{l|l}
a_{B^{(1)}}^{(1)}+a_{B^{(2)}}^{(2)}+\cdots+a_{B^{(m)}}^{(m)} & \begin{array}{c}
a^{(1)}, a^{(2)}, \cdots, a^{(m)} \in \mathbb{R}, \\
B^{(1)}, B^{(2)}, \cdots, B^{(m)} \in \mathbb{D}_{n-1}
\end{array}
\end{array}\right\} \cup\{0\}
$$

where 0 is the zero element of this abelian group. We define products in $\boldsymbol{D}_{1}$ in the set $\left\{a_{B} \mid a \in \mathbb{R}, B \in \mathbb{D}_{n-1}\right\}$ by

$$
a_{B} \cdot c_{D}:=a c_{B+D}, a, c \in \mathbb{R}, B, D \in \mathbb{D}_{n-1},
$$


and extend them to whole $\boldsymbol{D}_{n}$ by using the distributive law. Note that the addition $B+D$ at their dimensions has already been defined in $\mathbb{D}_{n-1}$. As in the case of Depth 1 , the element $1_{O}$, where $O$ is the zero element in $\mathbb{D}_{n-1}$ is the unit element for the multiplication in $\boldsymbol{D}_{n}$, and the element 0 is the zero element for the multiplication in $\boldsymbol{D}_{n}$. We have the following.

Lemma 1.4. $\left(\boldsymbol{D}_{n},+, 0, \cdot, 1_{O}\right)$ is a commutative ring.

To realize the equality $a_{C}+b_{C}=a+b_{C}, a, b \in \mathbb{R}, C \in \mathbb{D}_{n-1}$, we define an ideal in $\boldsymbol{D}_{n}$. Let $\boldsymbol{Z}_{n}$ be the additive subgroup of $\boldsymbol{D}_{n}$ generated by the elements in the set

$$
\left\{a_{B}+a_{B}^{\prime}-\left(a+a^{\prime}\right)_{B} \mid a, a^{\prime} \in \mathbb{R}, B \in \mathbb{D}_{n-1}\right\} \cup\left\{0_{B} \mid B \in \mathbb{D}_{n-1}\right\} .
$$

Then similarly as Lemma 1.2, we have the following.

Lemma 1.5. $\boldsymbol{Z}_{1}$ is an ideal in $\boldsymbol{D}_{1}$.

We define

$$
\mathbb{D}_{n}:=\boldsymbol{D}_{n} / \boldsymbol{Z}_{n}
$$

and we will always identify an element $A$ in $\boldsymbol{D}_{n}$ with the element $A+\boldsymbol{Z}_{n}$ in the residue ring $\mathbb{D}_{n}$. We call elements in $\mathbb{D}_{n}$ dimensioned numbers of depth $n$. For an element

$$
A=a_{B^{(1)}}^{(1)}+a_{B^{(2)}}^{(2)}+\cdots+a_{B^{(m)}}^{(m)}
$$

in $\boldsymbol{D}_{n}$, we call $a^{(1)}, a^{(2)}, \cdots$ heads of $A$ and $B^{(1)}, B^{(2)}, \cdots$ dimensions of $A$. We say a dimensioned number $A$ is simple if $A$ is of the form

$$
A=a_{B},
$$

namely, containing only one head. Otherwise, $A$ is called a compound dimensioned number. If the head of a simple dimensioned number $A$ is 1 , namely,

$$
A=1_{B},
$$

then we call $A$ monic.

Next we will introduce a linear order in $\boldsymbol{D}_{n}$. Let

$$
A=a_{B^{(1)}}^{(1)}+a_{B^{(2)}}^{(2)}+\cdots+a_{B^{(m)}}^{(m)} \in \mathbb{D}_{n}
$$

be a non-zero element. Without loss of generality, we may assume

$$
a^{(1)} \neq 0, a^{(2)} \neq 0, \ldots a^{(m)} \neq 0 \text { and } B^{(1)}>B^{(2)}>\cdots>B^{(m)} .
$$

Then we call $a_{b^{(1)}}^{(1)}$ the dominant term of $A$. We define the sign of $A$ according to that of the head of the dominant term:

$$
\begin{aligned}
& A>0(A \text { is positive }) \quad \Longleftrightarrow \quad a^{(1)}>0, \text { and } \\
& A<0(A \text { is negative }) \quad \Longleftrightarrow \quad a^{(1)}<0,
\end{aligned}
$$

and define a relation $>$ in $\mathbb{D}_{1}$ by

$$
A>B \Longleftrightarrow A-B>0, A, B \in \mathbb{D}_{1}
$$

Then we have the following.

Lemma 1.6. $\left(\mathbb{D}_{n},+, 0, \cdot, 1_{0},>\right)$ is an ordered integral domain. 
The proof is similar to that of Lemma 1.3.

Next, we will define a map $i_{n-1}^{n}: \mathbb{D}_{n-1} \rightarrow \mathbb{D}_{n}$ by

$$
i_{n-1}^{n}\left(a_{B^{(1)}}^{(1)}+a_{B^{(2)}}^{(2)}+\cdots+a_{B^{(m)}}^{(m)}\right):=a_{i_{n-2}^{n-1}\left(B^{(1)}\right)}^{(1)}+a_{i_{n-2}^{n-1}\left(B^{(2)}\right)}^{(2)}+\cdots+a_{i_{n-2}^{n-1}\left(B^{(m)}\right)}^{(m)},
$$

$a^{(1)}, a^{(2)}, \cdots, a^{(m)} \in \mathbb{R}, B^{(1)}, B^{(2)}, \cdots, B^{(m)} \in \mathbb{D}_{n-2}$. Note that the map $i_{n-2}^{n-1}: \mathbb{D}_{n-2} \rightarrow \mathbb{D}_{n-1}$ is already established.

Lemma 1.7. The map $i_{n-1}^{n}: \mathbb{D}_{n-1} \rightarrow \mathbb{D}_{n}$ is a well-defined injective ordered ring homomorphism.

Proof. To prove the well-definedness, it is sufficient to show that $i_{n-1}^{n}$ maps $\boldsymbol{Z}_{n-1}$ into $\boldsymbol{Z}_{n}$. Since

$$
\begin{aligned}
i_{n}^{n+1}\left(a_{B}+a_{B}^{\prime}-\left(a+a^{\prime}\right)_{B}\right) & =a_{i_{n-2}^{n-1}(B)}+a_{i_{n-2}^{n-1}(B)}^{\prime}-\left(a+a^{\prime}\right)_{i_{n-2}^{n-1}(B)} \in \boldsymbol{Z}_{n} \\
i_{n}^{n+1}\left(0_{B}\right) & =0_{i_{n-2}^{n-1}(B)} \in \boldsymbol{Z}_{n},
\end{aligned}
$$

$i_{n-1}^{n}$ maps $\boldsymbol{Z}_{n-1}$ into $\boldsymbol{Z}_{n}$. Hence it is well-defined. It is easy to see that this map is an ordered ring homomorphism. Finally, we will show the injectivity. Let $A=a_{B^{(1)}}^{(1)}+a_{B^{(2)}}^{(2)}+\cdots+a_{B^{(m)}}^{(m)} \in \mathbb{D}_{n-1}$ with $a^{(1)} \neq 0, a^{(2)} \neq 0, \ldots, a^{(m)} \neq 0$ and $B^{(1)}>B^{(2)}>\cdots>B^{(m)}$ and suppose that $i_{n-1}^{n}(A)=0$. Since $i_{n-2}^{n-1}$ is injective and order-preserving, we have $i_{n-2}^{n-1}\left(B^{(1)}\right)>i_{n-2}^{n-1}\left(B^{(2)}\right)>\cdots>i_{n-2}^{n-1}\left(B^{(m)}\right)$. Hence $i_{n-1}^{n}(A) \neq 0$. It is a contradiction.

Now we have constructed the rings $\mathbb{D}_{n}$ of dimensioned numbers of depth $n$ for all non-negative integers $n$ together with the injective order ring homomorphisms

$$
i_{n}^{n+1}: \mathbb{D}_{n} \rightarrow \mathbb{D}_{n+1}, n=0,1,2, \ldots
$$

Hence we have an inductive system $\left(\mathbb{D}_{n}, i_{n}^{n+1}\right)$ and accordingly an inductive limit

$$
\mathbb{D}=\lim _{\longrightarrow}\left(\mathbb{D}_{n}, i_{n}^{n+1}\right) .
$$

Then we have a commutative diagram

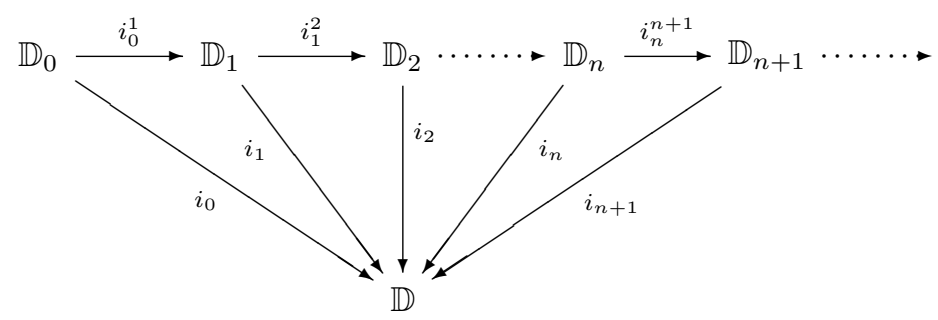

Here, $i_{n}: \mathbb{D}_{n} \rightarrow \mathbb{D}, n=0,1,2, \ldots$ is a natural map. Since all $i_{n}^{n+1}$ 's are injective, so are $i_{n}$ 's. From now on, we will identify an element $A \in \mathbb{D}_{n}$ with its image $i_{n}(A) \in \mathbb{D}$, and we often write $A$ instead of $i_{n}(A)$, and we call $A$ dimensioned number of depth $n$. Moreover, each map $i_{n}^{n+1}$ preserves the order $>$, and hence the inductive limit $\mathbb{D}$ naturally inherits it.

Theorem 1.8. ( $\mathbb{D},+, 0, \cdot, 1,>)$ is an ordered integral domain having the following properties:

(1) $a_{B}+a_{B}^{\prime}=\left(a+a^{\prime}\right)_{B}$

(2) $a_{B} \cdot c_{D}=a c_{B+D}$

(3) $A=A_{0}=A_{0_{B}}$ 
for $a, a^{\prime}, c \in \mathbb{R}$ and $A, B, D \in \mathbb{D}$.

The equality in (3) in the theorem means that if a dimension number contains numeral 0 at some depth, then we can ignore it together with the dimension hanging from the zero. We call this nullification. Nevertheless, we often write $A_{0}$ instead of $A$, when we want to emphasize that the numeral lies in the greatest depth and there are no numerals in deeper places.

Proof. The statement (1) is almost clear from the definition of $\mathbb{D}_{n}$.

The statement (2) is also clear from the definition of the product in $\mathbb{D}_{n+1}$ when both $B$ and $D$ lies in $\mathbb{D}_{n}$. Suppose that the depth of $B$ and $D$ are different, say the depth $m$ of $B$ is greater than the depth $n$ of $D$. Then, from the commutative diagram, we have

$$
\begin{aligned}
i_{m+1}\left(a_{B}\right) \cdot i_{n+1}\left(c_{D}\right) & =i_{m+1}\left(a_{B}\right) \cdot i_{m+1}\left(i_{m}^{m+1} \circ i_{m-1}^{m} \circ \cdots \circ i_{n}^{n+1}\left(c_{D}\right)\right) \\
& =i_{m+1}\left(a_{B}\right) \cdot i_{m+1}\left(c_{i_{m-1}^{m} \circ \cdots \circ i_{n-1}^{n}(D)}\right) \\
& =i_{m+1}\left(a_{B} \cdot c_{i_{m-1}^{m} \circ \cdots \circ i_{n-1}^{n}(D)}\right) \\
& =i_{m+1}\left(a c_{B+i_{m-1}^{m} \circ \cdots \circ i_{n-1}^{n}(D)}\right) .
\end{aligned}
$$

By ignoring maps, we have $a_{B} \cdot c_{D}=a c_{B+D}$. Hence (2) holds.

(3) Let $A \in \mathbb{D}_{n}$ and $B \in \mathbb{D}_{m}$. Then we have

$$
i_{m+1}\left(0_{B}\right)=i_{m+1}(0)=i_{0}(0)
$$

and hence

$$
i_{n+m+2}\left(A_{0_{B}}\right)=i_{n+1}\left(A_{0}\right)=i_{n}(A) .
$$

By ignoring maps, we have $A_{0_{B}}=A_{0}=A$.

Next, we introduce the third binary operation $\wedge$ called symmetric power to dimensioned numbers, which corresponds to a "symmetric power" in functions of a single variable and their correspondence will be discussed in Section 2.

Let $\mathbb{D}^{\times}$denote the set of non-zero elements in $\mathbb{D}$, namely, $\mathbb{D}^{\times}:=\mathbb{D} \backslash\{0\}$. We set

$$
\begin{aligned}
\widehat{\boldsymbol{D}}_{0}:= & \text { the free abelian group generated by the elements in } \\
& \left\{A_{1}^{(1)} \wedge A_{2}^{(1)} \wedge \cdots \wedge A_{n_{1}}^{(1)} \cdot A_{1}^{(2)} \wedge A_{2}^{(2)} \wedge \cdots \wedge A_{n_{2}}^{(2)} \cdots \cdots A_{1}^{(m)} \wedge A_{2}^{(m)} \wedge \cdots \wedge A_{n_{m}}^{(m)}\right. \\
& \left.\mid A_{i}^{(j)} \in \mathbb{D}^{\times} \text {for } i=1, \ldots, n_{j}, n_{j} \geq 1, j=1, \ldots, m, \quad m \geq 1\right\},
\end{aligned}
$$

where $A_{1}^{(j)} \wedge A_{2}^{(j)} \wedge \cdots \wedge A_{n_{j}}^{(j)}$ is understood as $A_{1}^{(j)}$ when $n_{j}=1$. We call elements of the form $A_{1}^{(1)} \wedge A_{2}^{(1)} \wedge \cdots \wedge A_{n_{1}}^{(1)}$. $A_{1}^{(2)} \wedge A_{2}^{(2)} \wedge \cdots \wedge A_{n_{2}}^{(2)} \cdot \cdots \cdot A_{1}^{(m)} \wedge A_{2}^{(m)} \wedge \cdots \wedge A_{n_{m}}^{(m)}$ power polynomials.

We define a multiplication in $\widehat{\boldsymbol{D}}_{0}$ by

$$
\begin{aligned}
& \left(A_{1}^{(1)} \wedge A_{2}^{(1)} \wedge \cdots \wedge A_{n_{1}}^{(1)} \cdot A_{1}^{(2)} \wedge A_{2}^{(2)} \wedge \cdots \wedge A_{n_{2}}^{(2)} \cdot \cdots \cdot A_{1}^{(m)} \wedge A_{2}^{(m)} \wedge \cdots \wedge A_{n_{m}}^{(m)}\right) \\
& \cdot\left(B_{1}^{(1)} \wedge B_{2}^{(1)} \wedge \cdots \wedge B_{n_{1}^{\prime}}^{(1)} \cdot B_{1}^{(2)} \wedge B_{2}^{(2)} \wedge \cdots \wedge B_{n_{2}^{\prime}}^{(2)} \cdot \cdots \cdot B_{1}^{\left(m^{\prime}\right)} \wedge B_{2}^{\left(m^{\prime}\right)} \wedge \cdots \wedge B_{n_{m^{\prime}}^{\left(m^{\prime}\right)}}^{(2)}\right) \\
& \quad=A_{1}^{(1)} \wedge A_{2}^{(1)} \wedge \cdots \wedge A_{n_{1}}^{(1)} \cdot \cdots \cdot A_{1}^{(m)} \wedge \cdots \wedge A_{n_{m}}^{(m)} \cdot B_{1}^{(1)} \wedge \cdots \wedge B_{n_{1}^{\prime}}^{(1)} \cdot \cdots \cdot B_{1}^{\left(m^{\prime}\right)} \wedge \cdots \wedge B_{n_{m^{\prime}}^{\prime}}^{\left(m^{\prime}\right)}
\end{aligned}
$$

for power polynomials and extend it to general elements by the distributive law as in the case of $\boldsymbol{D}_{1}$. Moreover, let the element 1 the unit element for this multiplication. It is easy to see the following. 
Lemma 1.9. $\left(\widehat{\boldsymbol{D}}_{0},+, 0, \cdot, 1\right)$ is a non-commutative ring.

Note that among the binary operations defined, the symmetric powers are prior to the other operations unless parentheses are placed. To make both the multiplication and the symmetric power commutative and to make the power be to the multiplication what the multiplication be to the addition, we will define a two-sided ideal $\widehat{Z}_{0}$ by

$\widehat{Z}_{0}:=$ the two-sided ideal of $\left(\widehat{\boldsymbol{D}}_{0},+, 0, \cdot, 1\right)$ generated by the elements in

$$
\begin{aligned}
& \left\{A \cdot B-B \cdot A \mid A, B \in \widehat{D}_{0}\right\} \\
& \cup\{A_{1} \wedge \cdots \wedge \overbrace{A_{i}}^{i} \wedge \ldots \wedge \overbrace{A_{j}}^{j} \wedge \cdots \wedge A_{n}-A_{1} \wedge \ldots \wedge \overbrace{A_{j}}^{i} \wedge \ldots \wedge \overbrace{A_{i}}^{j} \wedge \wedge A_{n} \\
& \left.\quad \mid 1 \leq i<j \leq n, A_{1}, \ldots, A_{n} \in \mathbb{D}^{\times}, n \geq 2\right\} \\
& \cup\left\{A_{1} \wedge \cdots \wedge A_{n} \wedge\left(B_{1} \cdot B_{2}\right)-A_{1} \wedge \cdots \wedge A_{n} \wedge B_{1} \cdot A_{1} \wedge \cdots \wedge A_{n} \wedge B_{2}\right. \\
& \left.\quad \mid A_{1}, \ldots, A_{n}, B_{1}, B_{2} \in \mathbb{D}^{\times}, n \geq 1\right\} \\
& \cup\left\{A_{1} \wedge \cdots \wedge A_{n} \wedge 1_{B} \wedge 1_{C}-A_{1} \wedge \cdots \wedge A_{n} \wedge 1_{B \cdot C} \mid A_{1}, \ldots, A_{n} \in \mathbb{D}^{\times}, B, C \in \mathbb{D}, n \geq 0\right\} \\
& \cup\left\{A_{1} \wedge \cdots \wedge A_{n} \wedge 1_{k}-\left(A_{1} \wedge \cdots \wedge A_{n}\right)^{k} \mid A_{1}, \ldots, A_{n} \in \mathbb{D}^{\times}, n \geq 1, k \geq 1\right\} \\
& \cup\left\{A_{1} \wedge \cdots \wedge A_{n} \wedge 1_{0}-1_{0} \mid A_{1}, \ldots, A_{n} \in \mathbb{D}^{\times}, n \geq 1\right\},
\end{aligned}
$$

and define a residue ring

$$
\widehat{\mathbb{D}}_{0}:=\widehat{\boldsymbol{D}}_{0} / \widehat{Z}_{0} .
$$

We call elements in $\widehat{\mathbb{D}}_{0}$ dimensioned numbers with symmetric powers of depth 0 and nesting level 0 . We will always identify an element $A \in \widehat{\boldsymbol{D}}_{0}$ with $A+\widehat{Z}_{0} \in \widehat{\mathbb{D}}_{0}$. From the construction, the following facts are obvious:

Lemma 1.10. We have the following equalities.

(1) $A \cdot B=B \cdot A, A, B \in \widehat{\mathbb{D}}_{0}$.

(2) $(A \cdot B) \cdot C=A \cdot(B \cdot C), A, B, C \in \widehat{\mathbb{D}}_{0}$.

(3) $A_{1} \wedge A_{2}=A_{2} \wedge A_{1}, A_{1}, A_{2} \in \mathbb{D}^{\times}$.

(4) $A_{1} \wedge \cdots \wedge A_{n} \wedge\left(B_{1} \cdot B_{2}\right)=A_{1} \wedge \cdots \wedge A_{n} \wedge B_{1} \cdot A_{1} \wedge \cdots \wedge A_{n} \wedge B_{2}, A_{1}, \ldots, A_{n}, B_{1}, B_{2} \in \mathbb{D}^{\times}$.

(5) $1_{B} \wedge 1_{C}=1_{B \cdot C}, B, C \in \mathbb{D}$.

(6) $A_{1} \wedge \cdots \wedge A_{n} \wedge 1_{k}=\left(A_{1} \wedge \cdots \wedge A_{n}\right)^{k}, A_{1}, \ldots, A_{n} \in \mathbb{D}^{\times}, k \geq 1$. In particular, $A_{1} \wedge \cdots \wedge A_{n} \wedge 1_{1}=A_{1} \wedge \cdots \wedge A_{n}, A_{1}, \ldots, A_{n} \in \mathbb{D}^{\times}$.

(7) $A_{1} \wedge \cdots \wedge A_{n} \wedge 1_{0}=1_{0}, A_{1}, \ldots, A_{n} \in \mathbb{D}^{\times}$.

Lemma 1.11. $\left(\widehat{\mathbb{D}}_{0},+, 0, \cdot 1\right)$ is a commutative ring.

Lemma 1.10 suggests that the relation between the multiplication and the addition is similar to that between the power and the multiplication. For example, $(4)$ is a distributive law between the power and the multiplication and (5) is similar to the equality $1_{B} \cdot 1_{C}=1_{B+C}, B, C \in \mathbb{D}$. Moreover, $1_{1}$ looks like the unit element and $1_{0}$ looks like the zero element for the symmetric power. However, dimensioned numbers with symmetric powers of nesting level 1 like $(2+3 \wedge 4) \wedge(5 \wedge 6+7 \wedge 8)$ do not belong to $\widehat{\mathbb{D}}_{0}$. We can construct dimensioned numbers with symmetric powers of positive nesting levels, but we omit it because we do not use them in this paper. Instead, we develop dimensioned numbers with symmetric powers of any depth, by induction on depth.

Suppose that we have constructed the ring $\widehat{\mathbb{D}}_{n}$ of dimensioned numbers with symmetric powers of depth $n, n=0,1,2, \ldots$. Then we let $\widehat{\boldsymbol{A}}_{n+1}$ be the free abelian group generated by the symbols

$$
a_{B}, a \in \mathbb{R}, B \in \widehat{\mathbb{D}}_{n}
$$


and define a multiplication by

$$
a_{B} \cdot c_{D}=a c_{B+D}, a, c \in \mathbb{R}, B, D \in \widehat{\mathbb{D}}_{n},
$$

for simple dimensioned numbers and extend it to the whole $\widehat{\boldsymbol{A}}_{n+1}$ by the distributive law. We define an additive subgroup $\widehat{\boldsymbol{B}}_{n+1}$ of $\widehat{\boldsymbol{A}}_{n+1}$ as the subgroup generated by the elements in the set

$$
\left\{a_{B}+a_{B}^{\prime}-\left(a+a^{\prime}\right)_{B} \mid a, a^{\prime} \in \mathbb{R}, B \in \widehat{\mathbb{D}}_{n}\right\} \cup\left\{0_{B} \mid B \in \widehat{\mathbb{D}}_{n}\right\} .
$$

Then similarly as Lemma 1.2 , we have the following.

Lemma 1.12. $\widehat{\boldsymbol{B}}_{n+1}$ is an ideal in $\widehat{\boldsymbol{A}}_{n+1}$.

Hence we can define a residue ring

$$
\widehat{\mathbb{A}}_{n+1}:=\widehat{\boldsymbol{A}}_{n+1} / \widehat{\boldsymbol{B}}_{n+1} .
$$

We will identify a real number $a$ with $a_{0}$ as before.

Lemma 1.13. $\left(\widehat{\mathbb{A}}_{n+1},+, 0, \cdot, 1\right)$ is a commutative ring.

Next, we will introduce powers between elements in $\widehat{\mathbb{A}}_{n+1}$. Let $\widehat{\mathbb{A}}_{n+1}^{\times}$denote the set of non-zero elements in $\widehat{\mathbb{A}}_{n+1}$. We set

$$
\begin{aligned}
\widehat{\boldsymbol{D}}_{n+1}:= & \text { the free abelian group generated by the elements in } \\
& \left\{A_{1}^{(1)} \wedge A_{2}^{(1)} \wedge \cdots \wedge A_{n_{1}}^{(1)} \cdot A_{1}^{(2)} \wedge A_{2}^{(2)} \wedge \cdots \wedge A_{n_{2}}^{(2)} \cdots \cdots A_{1}^{(m)} \wedge A_{2}^{(m)} \wedge \cdots \wedge A_{n_{m}}^{(m)}\right. \\
& \left.\mid A_{i}^{(j)} \in \widehat{\mathbb{A}}_{n+1}^{\times} \text {for } i=1, \ldots, n_{j}, n_{j} \geq 1, j=1, \ldots, m, \quad m \geq 1\right\},
\end{aligned}
$$

where $A_{1}^{(j)} \wedge A_{2}^{(j)} \wedge \cdots \wedge A_{n_{j}}^{(j)}$ is understood as $A_{1}^{(j)}$ when $n_{j}=1$. We define a multiplication in $\widehat{\boldsymbol{D}}_{n+1}$ totally similarly as that in $\widehat{\boldsymbol{D}}_{0}$ and consider an ideal $\widehat{Z}_{n+1}$ in $\widehat{\boldsymbol{D}}_{n+1}$ totally similarly as $\widehat{Z}_{0}$ in $\widehat{\boldsymbol{D}}_{0}$ (replace all $\mathbb{D}^{\times}$'s in the definition by $\widehat{\mathbb{A}}_{n+1}^{\times}$), and define a residue ring

$$
\widehat{\mathbb{D}}_{n+1}:=\widehat{\boldsymbol{D}}_{n+1} / \widehat{Z}_{n+1} \text {. }
$$

We call elements in $\widehat{\mathbb{D}}_{n+1}$ dimensioned numbers with symmetric powers of depth $n+1$ and nesting level 0 . We will always identify an element $A \in \widehat{\boldsymbol{D}}_{n+1}$ with $A+\widehat{Z}_{n+1} \in \widehat{\mathbb{D}}_{n+1}$.

We have a sequence of natural inclusion

$$
\widehat{\mathbb{D}}_{0} \subset \widehat{\mathbb{D}}_{1} \subset \widehat{\mathbb{D}}_{2} \subset \cdots
$$

and consequently an inductive limit

$$
\widehat{\mathbb{D}}:=\lim _{\longrightarrow} \widehat{\mathbb{D}}_{n}
$$

We call elements in $\widehat{\mathbb{D}}$ dimensioned numbers with powers of nesting level 0 . Note that the ring $\mathbb{D}$ of dimensioned numbers without powers is a subring of $\widehat{\mathbb{D}}_{0}$, and hence a subring of $\widehat{\mathbb{D}}$. We have the following theorem. The proof is similar to that of Lemma 1.10 .

Theorem 1.14. $(\widehat{\mathbb{D}},+, 0, \cdot, 1)$ is a commutative ring having the following properties for $m=1,2, \ldots$

(1) $A_{1} \wedge A_{2}=A_{2} \wedge A_{1}, A_{1}, A_{2} \in \widehat{\mathbb{A}}_{m}^{\times}$.

(2) $A_{1} \wedge \cdots \wedge A_{n} \wedge\left(B_{1} \cdot B_{2}\right)=A_{1} \wedge \cdots \wedge A_{n} \wedge B_{1} \cdot A_{1} \wedge \cdots \wedge A_{n} \wedge B_{2}, A_{1}, \ldots, A_{n}, B_{1}, B_{2} \in \widehat{\mathbb{A}}_{m}^{\times}$.

(3) $1_{B} \wedge 1_{C}=1_{B \cdot C}, B, C \in \widehat{\mathbb{D}}$.

(4) $A_{1} \wedge \cdots \wedge A_{n} \wedge 1_{k}=\left(A_{1} \wedge \cdots \wedge A_{n}\right)^{k}, A_{1}, \ldots, A_{n} \in \widehat{\mathbb{A}}_{m}^{\times}, k \geq 1$. In particular, $A_{1} \wedge \cdots \wedge A_{n} \wedge 1_{1}=A_{1} \wedge \cdots \wedge A_{n}, A_{1}, \ldots, A_{n} \in \widehat{\mathbb{A}}_{m}^{\times}$. 
(5) $A_{1} \wedge \cdots \wedge A_{n} \wedge 1_{0}=1_{0}, A_{1}, \ldots, A_{n} \in \widehat{\mathbb{A}}_{m}^{\times}$.

Note that these equalities also hold at any depth of a simple dimensioned number. For example,

$$
a_{A_{1} \wedge A_{2}}=a_{A_{2} \wedge A_{1}}, a \in \mathbb{R}, A_{1}, A_{2} \in \widehat{\mathbb{A}}_{m}^{\times} .
$$

\section{Correspondence BetWeEn Dimensioned numbers AND FUnCTiOns of A SingLe VARIABLE}

In this section, we will construct a map from the ring of dimensioned numbers into the ring of functions of a single variable, and show the compatibility of binary operations including the power introduced in Section 1. Moreover, we establish a symbolic substituting method, which corresponds to compositions of functions.

First, we will construct a map from the ring $\mathbb{D}$ of dimensioned numbers without powers into the ring of real-valued functions of $x$ with domain $(0,+\infty)$. The rules are simple. One is to correspond a real number $a$ to the constant function $f(x)=a$. The other is, if a dimensioned number $A$ corresponds to a function $f(x)$, then $1_{A}$ corresponds to $x^{f(x)}$. Additions and multiplications are preserved. For example,

$$
\begin{array}{cl}
1_{3} \mapsto x^{3}, & 2_{3}=2 \cdot 1_{3} \mapsto 2 x^{3}, \\
1_{1_{1}} \mapsto x^{x^{1}}=x^{x}, & 3_{2_{4}+1_{-3}} \mapsto 3 x^{2 x^{4}+x^{-3}} .
\end{array}
$$

Theorem 2.1. The above rules determine a well-defined homomorphism from $\mathbb{D}$ to the ring of functions of a single variable $x$ with domain $(0,+\infty)$.

Proof. We will prove by induction on depth.

For dimensioned numbers of depth 0 , the assertion is obvious.

Suppose that we have proved the assertion for dimensioned numbers of depth $n$. We will show the case for the depth $n+1$. For the proof, we must observe that two dimensioned numbers of the form $a_{B}+a_{B}^{\prime}$ and $\left(a+a^{\prime}\right)_{B}, a, a^{\prime} \in \mathbb{R}, B \in \mathbb{D}_{n}$ correspond to the same function with domain $(0,+\infty)$. If $B \mapsto f(x)$ and the domain of $f$ contains $(0,+\infty)$, then we have

$$
\begin{aligned}
a_{B}+a_{B}^{\prime} & \mapsto a x^{f(x)}+a^{\prime} x^{f(x)} \\
\left(a+a^{\prime}\right)_{B} & \mapsto\left(a+a^{\prime}\right) x^{f(x)}
\end{aligned}
$$

and hence the images coincide for $x \in(0,+\infty)$.

Next, we will see that $0_{B} \mapsto 0$. It is seen from the correspondence

$$
0_{B} \mapsto 0 \cdot x^{f(x)} .
$$

Finally we examine the compatibility of multiplications. Let $a, c \in \mathbb{R}$ and $B, D \in \mathbb{D}_{n}$ and suppose that $B \mapsto f(x)$ and $D \mapsto g(x)$ with domain $(0,+\infty)$. Then we have

$$
\begin{aligned}
a_{B} \cdot c_{D} & \mapsto a x^{f(x)} \cdot c x^{g(x)} \\
a c_{B+D} & \mapsto a c x^{f(x)+g(x)} .
\end{aligned}
$$

Since the images coincide for $x \in(0,+\infty)$, the multiplications are compatible.

Hence we have proved that the correspondence is a well-defined homomorphism.

Next we consider the correspondence between dimensioned numbers with symmetric powers and functions of a single variable. Note that, in this case, not all dimensioned numbers correspond to real-valued functions. The rule is, in addition to the above-mentioned ones,

$$
A_{1} \wedge A_{2} \wedge \cdots \wedge A_{n} \mapsto x^{\log _{x} f_{1}(x) \cdot \log _{x} f_{2}(x) \cdots \cdots \cdot \log _{x} f_{n}(x)},
$$


where $A_{i} \in \mathbb{A}_{m}^{\times}, m \geq 1$ with $A_{i} \mapsto f_{i}(x), i=1,2, \ldots, n$. The image in (5), which we call the symmetric power of $f_{1}(x), f_{2}(x), \ldots, f_{n}(x)$, is meaningful, if $f_{1}(x), f_{2}(x), \ldots, f_{n}(x)$ have a common interval $I \subset(0,+\infty)$ such that $f_{i}(x)>0, x \in I, i=1,2, \ldots, n$.

Theorem 2.2. Let $A \in \widehat{\mathbb{D}}$ and consider all symmetric powers appearing in $A$. We take the constituents $A_{1}, A_{2}, \ldots, A_{n} \in \mathbb{A}_{m}^{\times}$for some $m$ in such symmetric powers. Suppose the functions $f_{1}(x), f_{2}(x), \ldots, f_{n}(x)$ corresponding to $A_{1}, A_{2}, \ldots, A_{n}$ have a common interval $I \subset(0,+\infty)$ such that $f_{i}(x)>0, x \in I, i=$ $1,2, \ldots, n$. Then $A$ corresponds to a real-valued function with domain I. This correspondence is well-defined.

Proof. We will prove by induction on $m$ for which $A \in \widehat{\mathbb{D}}_{m}$. Since an element in $\widehat{\mathbb{D}}_{m}$ is a sum of the products of elements of the form $A=A_{1} \wedge A_{2} \wedge \cdots \wedge A_{n}$ where $A_{i} \in \mathbb{A}_{m-1}^{\times}$, it suffices to show the assertion for such $A$.

The case of $m=0$ : We take an element $A \in \widehat{\boldsymbol{D}}_{0}$ of the form

$$
A=A_{1} \wedge A_{2} \wedge \cdots \wedge A_{n},
$$

where $A_{i} \in \mathbb{D}^{\times}$for $i=1, \ldots, n$. From Theorem 2.1, we see that the function $f_{i}(x)$ corresponding to $A_{i}$ is defined on the interval $(0,+\infty)$ and positive on a common interval $I$ for $i=1, \ldots, n$ from the assumption. Hence from (5), $A$ corresponds to a function with domain $I$. If $B \in \widehat{\mathbb{D}}_{0}$ is a sum of the products of such simple symmetric powers like $A$ with a common domain $I$, then it is clear that $B$ corresponds to a function with domain $I$. Hence we proved that the correspondence is defined for all elements in $\widehat{\boldsymbol{D}}_{0}$. Next, we must observe that all elements in $\widehat{Z}_{0}$ correspond to constant 0 . It is straightforward and easy. For example, consider an element $A_{1} \wedge \cdots \wedge A_{n} \wedge 1_{B} \wedge 1_{C}-A_{1} \wedge \cdots \wedge A_{n} \wedge 1_{B \cdot C}, A_{1}, \ldots, A_{n} \in \mathbb{D}^{\times}, B, C \in \mathbb{D}, n \geq 0$. Suppose $A_{i} \mapsto f_{i}(x), i=1, \ldots, n, B \mapsto g(x)$ and $C \mapsto h(x)$. We have

$$
\begin{aligned}
A_{1} \wedge \cdots \wedge A_{n} \wedge 1_{B} \wedge 1_{C} & \mapsto x^{\log _{x} f_{1}(x) \cdots \cdots \log _{x} f_{n}(x) \cdot \log _{x} x^{g(x)} \cdot \log _{x} x^{h(x)}} \\
& =x^{\log _{x} f_{1}(x) \cdots \cdot \log _{x} f_{n}(x) \cdot g(x) \cdot h(x)} \\
A_{1} \wedge \cdots \wedge A_{n} \wedge 1_{B \cdot C} & \mapsto x^{\log _{x} f_{1}(x) \cdots \cdots \log _{x} f_{n}(x) \cdot \log _{x} x^{g(x) \cdot h(x)}} \\
& =x^{\log _{x} f_{1}(x) \cdots \cdot \log _{x} f_{n}(x) \cdot g(x) \cdot h(x)}
\end{aligned}
$$

Hence we have

$$
A_{1} \wedge \cdots \wedge A_{n} \wedge 1_{B} \wedge 1_{C}-A_{1} \wedge \cdots \wedge A_{n} \wedge 1_{B \cdot C} \mapsto 0 .
$$

So the correspondence is well-defined for elements in $\widehat{\mathbb{D}}_{0}$.

Suppose that we have proved well-definedness for elements in $\widehat{\mathbb{D}}_{m}$. Let $A \in \widehat{\mathbb{D}}_{m+1}$. The dimensioned number $A$ consists of the elements of the form $a_{B}, a \in \mathbb{R}, B \in \widehat{\boldsymbol{D}}_{0}$, connected by binary operations,$+ \times, \wedge$ at depth 0 . From the assumption of the induction, such $B$ 's correspond to functions that are defined on a common interval $I$, and hence so do $a_{B}$ 's. Similarly as in the case of $m=0$, we can deduce that $A$ corresponds to a real-valued function with domain $I$ from the assumption in this theorem. Hence we have proved well-definedness for $\widehat{\mathbb{D}}_{m+1}$.

If a dimension number $A$ corresponds to a function $f(x)$, then we will denote $A \sim f(x)$.

Corollary 2.3. Let $A, B \in \widehat{\mathbb{D}}$ and $A \sim f(x)$ and $B \sim g(x)$. Then we have the following.

(1) $A \wedge 1_{B} \sim f(x)^{g(x)}$.

(2) $c_{A} \wedge 1_{d}=c_{d A}^{d} \sim c^{d} x^{d f(x)}, c, d \in \mathbb{R}$.

(3) $1_{A} \wedge 1_{B}=1_{A \cdot B} \sim x^{f(x) g(x)}$.

The proof is immediate.

Next, we describe substitutions by using dimensioned numbers. Let $A \in \mathbb{D}, B \in \widehat{\mathbb{D}}$ and $A \sim f(x), B \sim g(x)$. We want to compute the composite function $f \circ g(x)$ in terms of $A$ and $B$. For this purpose, we introduce a natural decomposition of dimensioned numbers in $\mathbb{D}$. The definition is done by induction on the depth of a 
dimensioned numbers. For depth 0 dimensioned numbers, we leave them as they are, because they correspond to constant functions. We suppose the natural decomposition $\tilde{B}$ of $A \in \mathbb{B}_{n}$ is established. An element in $\mathbb{D}_{n+1}$ is a sum of simple dimensioned numbers of the form $a_{B}, a \in \mathbb{R}, B \in \mathbb{D}_{n}$. We rewrite this by $a \cdot 1_{1} \wedge 1_{B}$, and take the sum of such rewritten forms. It is the natural decomposition of an element in $\mathbb{D}_{n+1}$. For example, a dimensioned number of depth 1 has a natural decomposition like

$$
a_{b}+c_{d}=a \cdot 1_{1} \wedge 1_{b}+c \cdot 1_{1} \wedge 1_{d},
$$

and a dimensioned number of depth 2 has

$$
a_{b_{c}+d_{e}}=a \cdot 1_{1} \wedge 1_{\left(b \cdot 1_{1} \wedge 1_{c}+d \cdot 1_{1} \wedge 1_{e}\right)} .
$$

We have the following result.

Theorem 2.4. Let $A \in \mathbb{D}, B \in \widehat{\mathbb{D}}$ and $A \sim f(x), B \sim g(x)$. Then the composition $f \circ g(x)$ corresponds to the dimensioned number obtained by replacing each $1_{1}$, appearing in the natural decomposition of $A$, by $B$. We denote the dimensioned number after this operation by $\left.A\right|_{1_{1} \leftarrow B}$.

Proof. We will prove by induction on the depth of $A$.

If the depth is 0 , then $f(x)$ is a constant function, so there is nothing to prove.

Suppose we have shown the statement for $A \in \mathbb{D}_{n}$. Consider a dimensioned number $A$ whose depth is $n+1$. It is enough to show the statement for those $A$ of the form

$$
A=a_{C}, a \in \mathbb{R}, C \in \mathbb{D}_{n} .
$$

If $C \sim h(x)$, then

$$
A \sim f(x)=a x^{h(x)} .
$$

From the assumption of induction, we have

$$
\left.C\right|_{11 \leftarrow B} \sim h \circ g(x)
$$

If we replace $1_{1}$ in $A=a \cdot 1_{1} \wedge 1_{C}$ by $B$, then we have

$$
\begin{aligned}
& \left.A\right|_{1_{1} \leftarrow B}=a \cdot B \wedge 1_{\left.C\right|_{1_{1} \leftarrow B}} \\
& \sim a g(x)^{h \circ g(x)} \quad \text { (by Corollary 2.3(1)) } \\
& =f \circ g(x) \text {. }
\end{aligned}
$$

Hence the statement is valid for $n+1$.

For functions corresponding to monic dimensioned number, the result will be simpler.

Corollary 2.5. Let $A \in \mathbb{D}, B \in \widehat{\mathbb{D}}$ and $A \sim f(x), 1_{B} \sim g(x)$. Then the composition $f \circ g(x)$ corresponds to the dimensioned number obtained by replacing each $1_{1}$, appearing in the natural decomposition of $A$, by $1_{B}$, and hence replacing each $1_{1} \wedge 1_{D}(D \in \widehat{\mathbb{D}})$ by $1_{B \cdot D}$, .

We will show some examples.

Example 2.6. Let

$$
f(x)=2 x^{3 x^{4}} \sim 2_{3_{4}} .
$$


Then we have, from Theorem 2.4,

$$
\begin{aligned}
f \circ f(x) & \left.\sim 2 \cdot 1_{1} \wedge 1_{3 \cdot 1_{1} \wedge 1_{4}}\right|_{1_{1} \leftarrow 2_{3}} . \\
& =2 \cdot 2_{3_{4}} \wedge 1_{3 \cdot 2_{3_{4}}} \wedge 1_{4} \\
& =2 \cdot 2_{3_{4}} \wedge 1_{3 \cdot 2^{4} 3_{4} \cdot 4} \\
& =2 \cdot 2_{3_{4}} \wedge 1_{3 \cdot 16_{12_{4}}} \\
& =2 \cdot\left(2 \cdot 1_{3_{4}}\right) \wedge 1_{48_{12_{4}}} \\
& =2 \cdot 2 \wedge 1_{48_{12_{4}}} \cdot 1_{3_{4}} \wedge 1_{48_{12_{4}}} \\
& =2 \cdot 2 \wedge 1_{48_{12_{4}}} \cdot 1_{34_{4} \cdot 48_{12_{4}}} \\
& =2 \cdot 2 \wedge 1_{48_{12_{4}}} \cdot 1_{144_{4+12}} \\
& \sim 2^{1+48 x^{12 x^{4}}} \cdot x^{144 x^{4+12 x^{4}}} .
\end{aligned}
$$

Example 2.7. Let $f(x)$ be the same as in Example 2.6, and

$$
g(x)=x^{5 x^{4}-2 x^{-1}} \sim 1_{5_{4}-2-1} .
$$

Then we have, from Corollary 2.5,

$$
\begin{aligned}
& \left.f \circ g(x) \sim 2 \cdot 1_{1 \cdot 3 \cdot 1_{1 \cdot 4}}\right|_{1_{1} \leftarrow 1_{5_{4}-2}-1} . \\
& =2 \cdot 1_{\left(5_{4}-2-1\right) \cdot 3 \cdot 1_{\left(5_{4}-2_{-1}\right) \cdot 4}} \\
& =2_{\left(5_{4}-2-1\right)} \cdot 3_{20_{4}-8_{-1}} \\
& =215_{4+20} 0_{4}-8_{-1}-6{ }_{-1+20} 0_{-1} 8_{-1}
\end{aligned}
$$

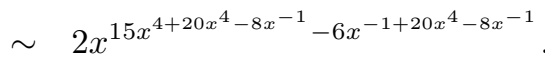

Comparing these two examples, we observe that the numerals in the result of Example 2.6, like 48 and 144, are influenced by the computations in deeper numerals, while those of Example 2.7, like 15 and -6, are just the result of computations of the numerals at the same depth. That is why monic dimensioned numbers are much easier to treat than non-monic ones.

\section{EXTENDED DIMENSIONED NUMBERS}

In this section we will introduce extended dimensioned numbers and show some examples.

Extended dimensioned numbers are dimensioned numbers which allow infinitely many numerals. There are three types of extended dimensioned numbers:

I. There are infinite sums or infinite products at some depths, but the total depth is finite. For example,

$$
1_{11_{1}-1_{2}+1_{3}-1_{4}+1_{5}-\cdots}
$$

II. There are only finitely many numerals at each depth, but the total depth is infinite. For example,

$$
1_{1+1_{1+2} 1_{1+3} 3_{1+4}+5} .
$$

III. There are infinite sums or infinite products at some depths, and the total depth is infinite. For example,

$$
1_{1+1_{-1}+1_{-1-1}+1_{-1_{-1}}+1_{-1_{-1} 1_{-1}}+\cdots}+\cdots
$$


The binary operations are defined similarly for extended dimensioned numbers, and the rule of substitution is also similar to Theorem 2.4 and Corollary 2.5. The difference between ordinary dimensioned numbers and extended dimensioned numbers are the correspondence to functions. We don't have standard ways to correspond extended dimensioned numbers with functions of a single variable, so we must make some interpretations of extended dimensioned numbers as functions. There may be several ways of interpretation, as the next example shows.

Example 3.1. Consider an extended dimensioned number

$$
A=1_{1_{1}} \ldots
$$

The simplest way of interpreting $A$ is to regard it as a limit of finite depth dimensioned numbers

$$
A_{n}=\underbrace{1_{1_{1} \cdot \ddots_{1}}}_{n} .
$$

Hence the interpretation of $A$ is

$$
f(x)=\lim _{n \rightarrow \infty} \underbrace{x^{x^{x^{.}}}}_{n} .
$$

In [RB, Chapter 7], it is proved that this limit exists for $e^{-e} \leq x \leq e^{1 / e}$ and is mutually inverse to the function

$$
g(x)=x^{1 / x}, e^{-1} \leq x \leq e .
$$

Another interpretation is, to regard $A$ as a solution of the functional equation

$$
A=1_{A} .
$$

If $A \sim y, y$ is a function of $x$, then we can rewrite above equation as

$$
y=x^{y} .
$$

Then $y$ is an implicit function of $x$. It is easy to see that there exists only one solution $y$ of this equation for each $x$ in $0<x \leq 1$, and two solutions for $x$ in $1<x<e^{1 / e}$. Hence $y$ is a two-valued function.

In this example, we can reproduce $A$ from the equation $A=1_{A}$ by successive substitution, namely, repeating substitution of $A=1_{A}$ into $A$ in the right-hand side of equation:

$$
A=1_{A}=1_{1_{A}}=1_{1_{1_{A}}} \cdots=1_{1_{1}} \cdot .
$$

Successive substitution must be done from smaller depth to greater depth. Hence successive substitution determines extended dimensioned numbers successively from the smaller depth.

\section{EXAMPLES OF SOLVING FUNCTIONAL EQUATIONS}

In this section we will solve some functional equations by using dimensioned numbers.

If an ordinary dimensioned number satisfies the equation, then it is a real solution, but we usually get extended dimensioned numbers as solutions, so they are just formal solutions and it is desirable to evaluate them from numerical point of view. Note that we will only search for monic solutions, because non-monic solutions are harder to treat as Examples 2.6 and 2.7 show. 


\section{Example 4.1.}

$$
f(x) f\left(x^{2}\right)=x^{x} \sim 1_{1_{1}}
$$

If we put

$$
f(x) \sim 1_{a_{b}+a_{b^{\prime}}^{\prime}}+\cdots,
$$

then

$$
f(x) f\left(x^{2}\right) \sim 1_{a_{b}}+2 a_{2 b}+a_{b^{\prime}}^{\prime}+2 a_{2 b^{\prime}}^{\prime}+\cdots
$$

Case 1: $a_{b}=1_{1}$. The solution is

$$
f_{1}(x) \sim 1_{1_{1}-2_{2}+4_{4}-8_{8}+\cdots,}
$$

which converges for $0<x<1$.

Case 2: $2 a_{2 b}=1_{1}$. The solution is

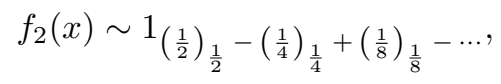

which converges for $0<x<\infty$.

These two solutions are numerically very good.

\section{Example 4.2.}

$$
y^{y^{y}}=x^{3} \sim 1_{3}
$$

If we put

$$
y \sim 1_{a_{b_{c}}}
$$

then

$$
\begin{aligned}
& y^{y^{y}} \sim 1_{a_{b_{c}}} \wedge 1_{1_{a_{b_{c}}} \wedge 1_{1_{a_{b_{c}}}}} \\
& =1_{a_{b_{c}} \cdot 1_{a_{b_{c}} \cdot 1_{a_{b_{c}}}}}
\end{aligned}
$$

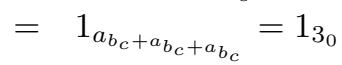

Hence we have

$$
a=3, b=-3, c=-3_{c}+3_{-3_{c}} .
$$

Applying successive substitution, we have:

$$
y=f(x) \sim 1_{3_{-3-3}-3} \cdot .^{+3-3} \cdot{ }^{+3-3} \cdot{ }^{+3-3} \cdot
$$

The dimensioned number $c$ is interpreted as the solution of the equation $c=-3 x^{c}+3 x^{-3 x^{c}}$. In fact, there is only one real solution $c$ of this equation for each $x>0$.

This solution is numerically very good. 


\section{Example 4.3.}

$$
f \circ f(x)=x^{x} \sim 1_{1_{1}}
$$

If we put

$$
f(x) \sim 1_{a_{b_{c}+b^{\prime}}^{\prime}+b_{c^{\prime \prime}}^{\prime \prime}+\cdots}, a, b \in \mathbb{R}, c \in \mathbb{D},
$$

then we can solve the equation. For convenience' sake, we put $f(x)$ in the following way by abuse of notations:

$$
f(x) \sim 1_{a_{b_{1}(x)+b_{2}(x)+b_{3}(x)+\cdots}},
$$

where $b_{i}(x), i=1,2, \ldots$ are functions of $x$. We have

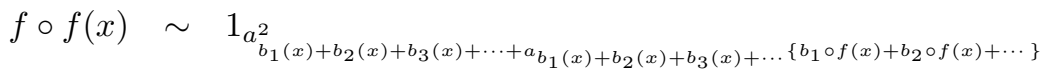

$$
\begin{aligned}
& =1_{1_{1}} \text {. }
\end{aligned}
$$

Hence $a^{2}=1$. We have two possibilities.

Case 1: $a=1$. Then we have

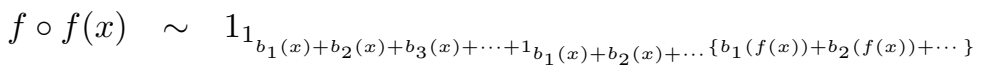

$$
\begin{aligned}
& =1_{1_{1}} \text {. }
\end{aligned}
$$

First, comparing the depth 2 of both sides, we put $b_{1}(x)=1$. Then we get an extra term

$$
1_{b_{1}(x)+b_{2}(x)+\cdots} \cdot b_{1}(f(x))=1_{1+b_{2}(x)+\cdots}
$$

To offset this, we put $b_{2}(x)=-1_{1+b_{2}(x)+\cdots}$. Then again, we get an extra term

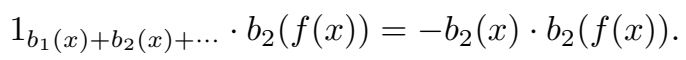

To offset this, we put $b_{3}(x)=b_{2}(x) \cdot b_{2}(f(x))$, and we repeat this:

$$
b_{n+1}(x)=b_{2}(x) b_{n}(f(x)), n=2,3, \ldots
$$

By induction, we can show

$$
b_{n+2}(x)=\prod_{i=0}^{n} b_{2}\left(f^{i}(x)\right), n=1,2, \ldots,
$$

where $f^{i}(x)$ is the $i$ times iteration of $f(x)$ and $f^{0}=\mathrm{id}$.

Observe that

$$
\begin{aligned}
x^{-b_{2}(x)} & \sim 1_{1_{1+b_{2}(x)+b_{3}(x)+\cdots}} \\
& =f(x) .
\end{aligned}
$$

Hence we have

$$
b_{2}(x)=-\log _{x} f(x)
$$

Substituting $f(x)$ into $x$ in $(7)$, we have

$$
\begin{aligned}
f(x)^{-b_{2}(f(x))} & =f^{2}(x) \\
& =x^{x}
\end{aligned}
$$


Next, raise to the $-b_{2}(f(x))$ power on both sides in (7), we have

$$
x^{b_{2}(x) b_{2}(f(x))}=f(x)^{-b_{2}(f(x))} .
$$

By (6) and (9), we have

$$
x^{b_{3}(x)}=x^{x}
$$

Taking logarithm,

$$
b_{3}(x)=x .
$$

Similarly we have

$$
\begin{aligned}
b_{4}(x) & =b_{3}(x) b_{2}\left(f^{2}(x)\right) \\
& =-x \cdot \log _{x}\left(f^{3}(x)\right) \\
& =-x \cdot \log _{x}\left(f(x)^{f(x)}\right), \\
b_{5}(x) & =b_{3}(x) b_{2}\left(f^{2}(x)\right) b_{2}\left(f^{3}(x)\right) \\
& =b_{3}(x) b_{3}\left(f^{2}(x)\right) \\
& =x \cdot x^{x}, \\
b_{6}(x) & =b_{5}(x) b_{2}\left(f^{4}(x)\right) \\
& =-x \cdot x^{x} \cdot \log _{x}\left(f(x)^{f(x)^{1+f(x)}}\right), \\
b_{7}(x) & =b_{5}(x) b_{2}\left(f^{4}(x)\right) b_{2}\left(f^{5}(x)\right) \\
& =x \cdot x^{x} \cdot x^{x^{1+x}} .
\end{aligned}
$$

Consequently,

$$
b_{n}(x)= \begin{cases}f^{0}(x) \cdot f^{2}(x) \cdots \cdots f^{2 m-2}(x) & n=2 m+1 \\ -f^{0}(x) \cdot f^{2}(x) \cdots \cdot f^{2 m-4}(x) \cdot \log _{x}\left(f^{2 m-2}(f(x))\right) & n=2 m .\end{cases}
$$

Hence we have, if we take $y=f(x)$,

$$
\begin{aligned}
y & \sim 1_{1_{b_{1}(x)+b_{2}(x)+b_{3}(x)+\cdots}} \\
& \sim x^{x^{1+x+x \cdot f^{2}(x)+x \cdot f^{2}(x) \cdot f^{4}(x)+\cdots} \cdot y^{-1} \cdot f^{2}(y)^{-x} \cdot f^{4}(y)^{-x \cdot x^{x}} \cdots}
\end{aligned}
$$

Even times iterates of $f$ are known functions, so that this expression admits successive substitutions. Hence we have obtained a formal solution.

Case 2: $a=-1$. By similar computations, we have

$$
\begin{aligned}
y & \sim 1_{-1_{b_{1}(x)+b_{2}(x)+b_{3}(x)+\cdots},} \\
& \sim x^{-x^{1+x+x \cdot f^{2}(x)+x \cdot f^{2}(x) \cdot f^{4}(x)+\cdots} \cdot y^{-1} \cdot f^{2}(y)^{-x} \cdot f^{4}(y)^{-x \cdot x^{x}} \cdots}
\end{aligned}
$$

We should find good interpretations for these two formal solutions.

\section{REFERENCES}

[Iz1] Izumi, H., Formal solutions of iterative functional equations, preprint.

[Iz2] Izumi, H., Analytic Solutions of Iterative Functional Equations, Talk at "Formal and Analytic Solutions of Differential and Difference Equations", 2011, Poland. http://www.impan.pl/ fasde/presentations/Izumi.pdf

[RB] Rajwade, A. R., Bhandari, A. K., Surprises and counterexamples in real function theory. Texts and Readings in Mathematics, 42. Hindustan Book Agency, New Delhi, 2007. 